\title{
Juvenile systemic lupus erythematosus onset patterns in Vietnamese children: a descriptive study of 45 children
}

Nguyen Thi Ngoc Dung ${ }^{1}$, Huynh Thoai Loan ${ }^{1}$, Susan Nielsen ${ }^{2}$, Marek Zak ${ }^{2 *}$ and Freddy K Petersen ${ }^{2}$

\begin{abstract}
Background: Incidence and disease pattern of childhood-onset SLE is reported to differ among ethnic groups.

Methods: To describe disease pattern and 6 month follow-up in a referral based cohort of 45 Vietnamese children with SLE. Forty-five children who were subsequently diagnosed to have systemic lupus erythematosus $(f / m=4 / 1)$ were referred to the Ho Chi Minh City Children's Hospital No.1 during a 12-month period in 2009.

Results: The mean age at diagnosis was 12.8 years $(S D=2.5)$. Thirty-seven (82\%) fulfilled criteria for lupus nephritis (LN). At diagnosis, impressively high SLEDAI and ECLAM scores were recorded (mean and SD), 23.8 (11.6) and 6 (2.3), respectively. The mean renal SLEDAI score was 8.2. The mean haemoglobin ( $\mathrm{g} / \mathrm{dL}, \mathrm{SD})$ was 8.5 (2.1). The Coombs test was positive in 30 of 36 children (83\%). The mean plasma creatinine was 0.98 (SD 1.2) and mean Westergren sedimentation rate was 83.6 (SD 37.4). The patient age at diagnosis was positively correlated to the SLEDAI $(p=0.034)$ and ECLAM ( $p=0.022)$. At 6 month follow-up of the 45 children, 15 patients were in complete remission, 5 were in partial remission, 6 had stable disease, 3 had relapsed, 3 had evolving disease, 2 had ongoing resistant disease and 4 had died. Seven patients were lost to follow-up. A second renal biopsy showed an improved ISN class in 13 of 15; in 2 cases the ISN class remained unchanged.

Conclusions: Forty-five Vietnamese children with SLE were referred to Ho Chi Minh Children's Hospital No. 1 during a16 month period from 2008-2009. These patients had a strikingly high prevalence of Coombs positive anaemia, a high prevalence of lupus nephritis, and very high SLEDAI and ECLAM scores at the time of diagnosis. While there may be referral biases, our Vietnamese SLE patients appear to have severe disease upon presentation but do reasonably well in the short-term.
\end{abstract}

\section{Background}

Fifteen to twenty percent of all systemic lupus erythematosus (SLE) patients have a disease onset before the age of 16 years. Childhood onset SLE is an unusual autoimmune disease with high risk of severe morbidity and mortality [1]. Incidence and disease patterns do differ among ethnic groups. It has been established that nonCaucasians have a higher incidence than Caucasians, and that renal disease is more frequent in nonCaucasian SLE children. Disease activity and damage, however, are primarily associated with major organ disease independent of the patient's ethnicity [2-4]. The

\footnotetext{
* Correspondence: rh16751@rh.dk

2Paediatric Rheumatology Unit, University Clinic of Paediatrics, Rigshospitalet, Blegdamsvej 9, Copenhagen DK-2100 Ø, Denmark

Full list of author information is available at the end of the article
}

objective of the current study was to investigate disease patterns at the time of diagnosis in a referral-based consecutive cohort of 45 Vietnamese children diagnosed with SLE at Ho Chi Minh Children's Hospital No. 1 and to perform a 6-month follow-up with emphasis on mortality and lupus nephritis (LN).

\section{Methods \\ Diagnosis}

Forty-five consecutive Vietnamese children with SLE participated in this prospective registration study. All new cases of childhood-onset SLE referred during the period from July 2008 to November 2009 to the tertiary Ho Chi Minh City (HMC) Children's Hospital No. 1 were included. The children were referred either from rural provinces, from the southern part of Vietnam, or 
from local hospitals in HMC. The American College of Rheumatology (ACR) SLE criteria were used for the diagnosis [5]. At the time of diagnosis, symptoms and biochemical parameters were recorded and used for SLE disease activity scores. These included the SLE Disease Activity Index (SLEDAI) and European Consensus Lupus Activity Measurement (ECLAM) [6-8].

The SLEDAI is a validated disease activity measure for childhood onset SLE with a total score of $0-105$. The tool consists of 24 weighted items grouped into following 9 domains: central nervous system (CNS), vascular, renal, musculoskeletal, serosal, dermal, immunologic, constitutional and hematologic. Renal disease activity was measured by the SLEDAI renal domain items [1]. ECLAM consists of 34 items grouped into 12 domains (9 clinical manifestations and 3 laboratory assessments) and measures active and evolving disease activity. ECLAM scores ranges from 0 to 10 .

All the SLEDAI and ECLAM scoring was done by one author (ND). Renal biopsies at diagnosis were done in suspicion of severe nephritis and evaluated according to the ISN (International Society of Nephrology) lupus nephritis grading system by an independent pathologist [9]. A follow-up biopsy after initial treatment was done whenever possible.

The following clinical symptoms and parameters were recorded: general symptoms (fatigue, pain and discomfort, general malaise), fever, malar rash, discoid rash, photosensitivity, alopecia, mouth ulcerations, vasculitis, nephritis, CNS symptoms (headache, seizures, psychosis, visual symptoms, cranial nerve manifestations and other possible neurological manifestations), myositis, serositis, gastrointestinal symptoms and pulmonary symptoms.

The blood tests performed were: haemoglobin, white blood cell count (WBC) and differential count, platelet count, erythrocyte sedimentation rate (ESR), C-reactive protein, serum creatinine, blood urea nitrogen, albumen, a 24-hour urinary protein excretion, red and white casts in urine, lactate dehydrogenase, creatinine kinase, glutamicoxaloacetic transaminase, and thyroid parameters. Other tests more specific for SLE were done including a Coombs test, $\mathrm{C} 3$ and $\mathrm{C} 4$ complement levels, the antinuclear antibodies (ANA), anti-DNA, and the anticardiolipin antibodies IgG and IgM. The ANA and anti-DNA levels were assessed using ELISA. Furthermore, blood pressure, heart rate, height, and weight were recorded. The local scientific committee approved the study.

\section{Treatment}

Induction treatment was administered according to the local guidelines, which were as follows.

1) All cases with CNS involvement or lupus nephritis ISN class IV or class III (with any symptoms: nephrotic syndrome, hypertension, elevated serum creatinine concentration, renal biopsy with 40 to 50 percent of glomeruli affected and/or crescent) were treated with:

a) Intravenous methylprednisolone $(1 \mathrm{~g} / 1.73 \mathrm{~m} 2 /$ day x 3-6 days, with a maximum dose of $1 \mathrm{~g}$ per treatment) and monthly intravenous

b) Intravenous cyclophosphamide (Суc) $(1 \mathrm{~g} / \mathrm{m} 2 /$ month; maximum single Cyc dose $0.5 \mathrm{~g}) \times 6$ consecutive months;

c) Oral prednisolone $1-2 \mathrm{mg} / \mathrm{kg} /$ day reduced according to disease activity.

2) Maintenance therapy consisted of azathioprine $(1-2 \mathrm{mg} / \mathrm{kg} /$ day $)$ and oral prednisolone. Additional treatment with an ACE inhibitor was administered to the majority of nephritis patients. All other cases or cases with mild nephritis (Class I, II, mild III) were treated with systemic steroid only.

\section{Renal outcome measures}

A renal biopsy at diagnosis was done if proteinuria exceeded $1 \mathrm{~g} / 24$ hours. Biopsy at 6 month followup was performed only in the nephritis cases treated with cyclophosphamide.

Renal outcome measures was in accordance with the local clinical practice classified as:

1) Complete remission (CR): If presence of normal serum creatinine, improvement in C3 level, proteinuria $<100 \mathrm{mg} / \mathrm{m} 2 /$ day or $<1+$ on urinary dipstick and/or inactive urinary sediment;

2) Partial remission (PR): If stabilization or improvement in serum creatinine level, improvement in C3 level, persistent reduction of proteinuria or, if in the nephrotic range at baseline, a decrease $>50 \%$ decrease in proteinuria and renal protein excretion $<50 \mathrm{mg} / \mathrm{kg} /$ day, if in the non-nephrotic range at baseline, a decrease $<50 \%$ of the pre-treatment value but renal protein excretion not more than $100 \mathrm{mg} / \mathrm{m} 2 /$ day, and reduction in active urinary casts;

3) Nonresponse (NR) If deterioration of renal function occurs exclusive of other causes, increase in proteinuria or reduction in proteinuria but not to the extent of CR or PR. At the 6 month follow-up CR or $\mathrm{PR}$ was regarded as favourable outcome.

4) A poor outcome was present in case of stable disease $(\mathrm{SD})$, resistant disease (RD), evolving disease (ED), relapse (RE) or death (MO).

\section{Results}

The mean age at SLE diagnosis was 12.8 years $(\mathrm{SD}=2.5)$. The female/male ratio was 4/1. All children were of 
Vietnamese origin. Table 1 displays demographics and disease characteristics at the onset of the SLE. The mean interval from initial symptoms to the SLE diagnosis was 23 weeks $(\mathrm{SD}=58)$. Upon exclusion of 3 outlier patients, the interval was reduced to a mean of 7.8 weeks $(\mathrm{SD}=9)$.

The most frequent initial SLE symptoms were: rash, fever, edema and arthritis. The group as a whole had a mean SLEDAI and ECLAM scores of $23.8(\mathrm{SD}=11.6)$ and $6(\mathrm{SD}=2.3)$, respectively, and a mean renal SLEDAI score of 8.2. Thirty-three of the 45 children fulfilled the criteria for lupus nephritis (LN), of which 29 had a renal biopsy. ISN Class IV was found in 20 cases and ISN Class III in 8 cases, one case had complex presentation with ISN Class III and V simultaneously. Nephritis was more prevalent in the children $<12$ years of age at the time of the SLE diagnosis. This finding was, however, not statistically significant (Fisher's Exact Test $\mathrm{p}=0.06$ ). Seventy percent of the children had a serum complement (C3 or C4) level below normal. C3 was below normal in $42 \%$ and $\mathrm{C} 4$ was below normal in $70 \%$ of the $\mathrm{LN}$ cases.

Elevated blood pressures were found in 20 of 45 patients primarily in LN patients. Hypertension was defined as systolic and/or diastolic blood pressure above the 95th percentile for gender and age. CNS involvement was found in 6 cases (13\%). Serositis was found in 16 $(36 \%)$ cases. Haemolytic anaemia with a positive Coombs test was found in $12 \%$. Overall, haematological abnormalities were found in $81 \%$ of the children at presentation. Forty-three patients (96\%) had an elevated

Table 1 Demographics and characteristic at disease onset in $\mathbf{4 5}$ Vietnamese children with SLE

\begin{tabular}{lcc}
\hline Parameter & \multicolumn{2}{c}{ Value } \\
\hline Number of patients & \multicolumn{2}{c}{45 12.1 years } \\
Female: male & \multicolumn{2}{c}{ Absent n } \\
Median age at diagnosis & Present n (\%) & 2 \\
Disease characteristics at diagnosis & $43(95)$ & 8 \\
Anti-DNA & $37(82)$ & 10 \\
Nephritis & $35(78)$ & 15 \\
Hematology & $30(67)$ & 15 \\
Malar rash & $30(67)$ & 19 \\
ANA & $26(58)$ & 21 \\
Arthritis & $24(53)$ & 28 \\
Photosensitivity & $17(38)$ & 29 \\
Ulcers & $16(36)$ & 38 \\
Serositis & $7(16)$ & 39 \\
CNS & $6(13)$ & 15 \\
Discoid changes & $30(67)$ & \\
Renal biopsy & &
\end{tabular}

antidsDNA. Mean erythrocyte sedimentation rate (ESR) was $83.6(\mathrm{SD}=37.4$, normal $0-20 \mathrm{~mm} /$ hour $)$.

At a 6-month follow-up of the 33 renal patients, 15 patients were in CR, 5 in PR, 6 had SD, 3 had ED, 2 had $\mathrm{RD}, 3$ had RE and $4 \mathrm{MO}$. Seven patients were lost to follow-up. Fifteen had a second renal biopsy after the course of 6 Cyc treatments. In 13 cases the ISN class had improved and in 2 cases it remained unchanged. In the first six months, 4 of the original 45 patients were know to have died, 2 before initiating treatment ( 1 with circulatory collapse and 1 of undiagnosed pneumonia), and 2 of tuberculosis shortly after initiating Cyc treatment.

Baseline parameters from the time of the diagnosis were investigated in order to look for predictors of short-term disease outcome. A low neutrophil count at the time of the diagnosis was associated with poor disease outcome $(p=0.01)$. The patient age at the time of the diagnosis was positively correlated to the SLEDAI $(\mathrm{p}=0.034)$ and ECLAM ( $p=0.022)$, which was attributed to a wider range of organ involvement in the oldest children (Figure 1).

\section{Discussion}

In a prospective study, all new cases of SLE referred to HCMC Children's Hospital No. 1 during a 16 months period were investigated. The ratio female/male of $4 / 1$ was in accordance with the literature. However, a number of findings differed somewhat from previously published reports on childhood-onset SLE. First, the relatively high mean age of 12.8 years at the time of the diagnosis was unusual. This finding could be explained by the fact that no children below the age of 5 years were included, what could be a true pattern but is more likely a result of underreporting/lack of diagnosis. An

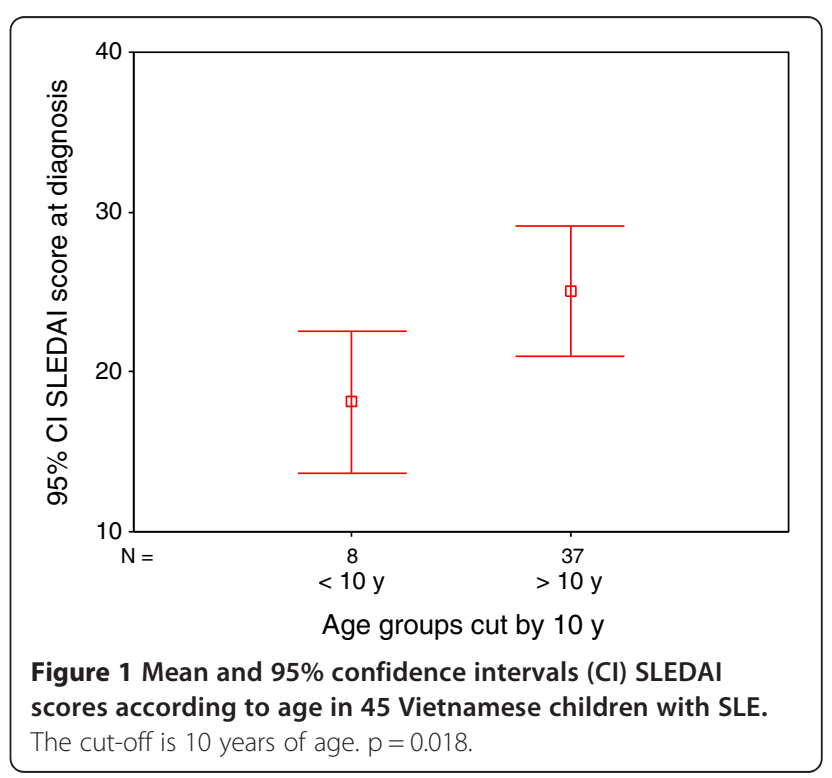


Italian group found that young children below 2 years of age at onset had very unspecific symptoms as rash and fever, making diagnosis more difficult [10]. In Vietnam, where infectious diseases are one of the major reasons of morbidity and mortality in young children, the diagnosis of SLE might prove particularly difficult. The mean age at diagnosis of childhood-onset SLE has been described to be different among ethnic groups, and is reported to be lower among non-Caucasian versus Caucasians [3,4,11-13].

SLEDAI and ECLAM have been validated as measures of disease activity for evaluation of response to therapy in SLE. ECLAM seems to be slightly superior to SLEDAI in measuring change over time, while SLEDAI seems to be slightly superior to ECLAM in measuring accumulated disease activity as a predictor of damage [6-8]. In general, at the time of referral children often have more severe disease activity with higher SLEDAI scores than adults $[1,10,14]$. The disease patterns for the current study showed remarkably sick children with a mean SLEDAI of 23.8 and a moderately high SLEDAI renal score. It remains to be investigated whether SLEDAI and/or ECLAM exhibit sufficient construct validity, accuracy and responsiveness in follow-up studies of SLE.

One of the major contributors to the high SLEDAI score was the $75 \%$ proportion of LN cases. This finding was in accordance with the literature $[1,3,10,12,13]$. All LN cases fulfilled criteria of nephritis according to the SLE criteria and biopsy was done in $89 \%$, the remaining not being suspected of having severe nephritis. As described by others, class IV was the most dominant feature on biopsy [1,3,10,11,14-18]. Of all biopsies from nephritis cases, $61 \%$ were classified as ISN class IV and $24 \%$ as ISN class III. No cases of class I - II was found in the initial biopsy, which could be explained by the fact that biopsies were done in suspected severe SLE nephritis cases only.

Hypertension was found in 20 of 45 . Hypertension at the time of the diagnosis was not predictive of the shortterm renal outcome. All class IV cases were treated according to the same protocol including induction therapy with low-dose Cyc and systemic steroids in 6 consecutive infusions 1 month apart. Follow-up biopsy $(\mathrm{n}=13)$ after 6 months showed marked improvement in 11 from class IV to III $(n=7)$ and class II $(n=4)$, in 2 slightly improvement among class III. Normalization of the $\mathrm{C} 3$ and $\mathrm{C} 4$ complements and reduction of proteinuria by greater than $25 \%$ by week 8 has been showed to strongly predict a good renal response to the SLE treatment [19]. Despite our cohort having a very high renal SLEDAI score at diagnosis, the majority of the nephritis patients showed significant improvement at 6 months followup. The short follow-up did not allow prediction of renal survival time, although the intensive treatment might prove predictive of a favourable renal outcome in comparison with results from other studies $[11,16-18,20]$. As childhood onset SLE is a chronic disease with exposure to lupus flares over many years, it will be interesting to see if there is a prognostic benefit to this early response to therapy in our renal patients in an extended prospective followup.

The most remarkable haematogical finding was a very high percentage of Coomb's positivity (83\%) with or without haemolyctic anaemia. The laboratory technique used was the slide rather than the tube method. The technique is well standardized and unlikely to yield false positive results. An explanation for this phenomenon could be the high incidence of infections in Vietnamese children, which in some instances may be followed by a positive Coombs test.

Central nervous system disease, defined as neurological involvement plus seizures and psychosis, was found in $13 \%$ of cases. Others have described an incidence of CNS disease from $7.7 \%$ to $45 \%$, with infantile SLE having the highest incidence and a trend of a greater incidence of CNS involvement in Afro-Americans than in Asian $[10,14,20,21]$.

Cardio-pulmonary disease was detected in $35 \%$ of the children, which is in accordance with the lupus literature, except for the Italian group who found a very high incidence in infantile SLE (approximately 75\%), and for Gedalia et al., who found a higher incidence in Afro-Americans compared to Latin-Americans [10,12,14,20,22].

Malar rash was observed in $66 \%$ and discoid lupus in $13 \%$, what was somewhat different from the literature and may be different among ethnic groups $[10,12,14,18,20,22]$. Gedalia et al. found a higher frequency of malar rash and discoid rash among Afro-Americans than LatinoAmericans. Our figures resemble what was found in AfroAmericans [22].

The very high percentage of positive anti-DNA (95\%) is a well known feature of SLE. Very few investigations of anti-phospholipid antibodies were done $(\mathrm{N}=10), 3$ IgM positive; 8 IgG positive. No coagulation disturbances were detected. Almost none had investigation of thyroid hormones due to economic reasons.

One patient died prior to treatment in a picture of circulatory collapse shortly after diagnosis without evidence of a bleeding disorder or intravascular dehydration. This may have been a case of SLE carditis, though this is speculative. Jakes et al. found deaths due to cardiovascular involvement in 6-40\% in the Asia-Pacific region [3].

One child died after a severe pneumonitis, with no microorganism identified, perhaps due to SLE lung involvement. Two patients died of TB. Both were diagnosed with pulmonary TB after the first infusion of cyclophosphamide and were on high dose corticosteroids. Chest $\mathrm{x}$-ray before treatment had been normal. However, no other TB testing was done. 
Only a few studies exist on the incidence and prevalence of SLE in South-East Asia. In the available literature from the region, the reported incidence rates range from 0.9 to 3.1 per 100.000 children [3]. The population in Southern Vietnam below 16 years of age is estimated to be approximately 10 million. Assuming that all new childhood SLE patients from South Vietnam were referred to HCM Children's Hospital No. 1 during the study period, a minimum incidence of approximately $0.4 / 100,000$ children might be calculated. However, the paediatricians at HMC Children's Hospital most likely only get to see the top of the iceberg. The true incidence and prevalence may be considerably higher for several reasons including under-reporting of the disease, the diagnostic challenges of SLE, and selection bias.

\section{Conclusions}

We report a cohort of 45 newly diagnosed SLE children from the Southern part of Vietnam. The disease pattern resembles in many ways the clinical patterns described by others of childhood onset SLE. However, the cohort did differ by demonstrating a very severe disease activity with very high SLEDAI scores and high SLEDAI renal scores at diagnosis. Although the short time follow-up of 6 months showed favourable histological treatment response, the data do not allow prediction of long time patient and renal survival. We plan an additional follow-up study that might answer some of these questions.

\section{Competing interests}

The authors declare that they have no competing interests.

\section{Authors' contribution}

All authors read and approved the final manuscript. NTND has participated in data collection and writing of the paper, HTL has participated in data collection and writing of the paper, NS has participated in statistical analysis and writing of the paper, ZM participated in statistical analysis and writing of the paper, PFK participated with the idea behind the study and writing of the paper.

\section{Author details}

'Department of Nephrology, Children's Hospital 1, 341 Su Van Hanh St., Ho Chi Minh City Dist 10, Vietnam. ${ }^{2}$ Paediatric Rheumatology Unit, University Clinic of Paediatrics, Rigshospitalet, Blegdamsvej 9, Copenhagen DK-2100 Ø, Denmark.

Received: 3 July 2012 Accepted: 10 November 2012

Published: 19 November 2012

\section{References}

1. Brunner HI, Gladman DD, Ibanez D, Urowitz MD, Silverman ED: Difference in disease features between childhood-onset and adult-onset systemic lupus erythematosus. Arthritis Rheum 2008, 58(2):556-562.

2. Hiraki LT, Feldman CH, Liu J, Larcón GS, Fisvher MA, Winkelmayer WC, Costenbader KH: Prevalence, Incidence, and demographics of systemic lupus erythematosus and lupus nephritis from 2000 to 2004 among children in the US Medicaid beneficiary population. Arthritis Rheum 2012 64(8):2669-2676.

3. Jakes RW, Bae SC, Louthren W, Mok CC, Navarra SV, Kwon N: Systematic review of the epidemiology of systemic lupus erythematosus in the Asia-Pacific region: prevalence, incidence, clinical features, and mortality. Arthritis Care Res (Hoboken) 2012, 64(2):159-168.
4. Hiraki LT, Benseler SM, Tyrrell PN, Harvey E, Hebert D, Silverman ED: Ethnic differences in pediatric systemic lupus erythematosus. J Rheumatol 2009, 36(11):2539-2546.

5. Tan EM, Cohen AS, Fries JF, Masi AT, McShane DJ, Rothfield NF, et al: The 1982 revised criteria for the classification of systemic lupus erythematosus. Arthritis Rheum 1982, 25(11):1271-1277.

6. Brunner HI, Silverman ED, Bombardier C, Feldman BM: European Consensus Lupus Activity Measurement is sensitive to change in disease activity in childhood-onset systemic lupus erythematosus. Arthritis Rheum 2003, 49(3):335-341

7. Ruperto N, Ravelli A, Cuttica R, Espada G, Ozen S, Porras O, et al: The Pediatric Rheumatology International Trials Organization criteria for the evaluation of response to therapy in juvenile systemic lupus erythematosus: prospective validation of the disease activity core set. Arthritis Rheum 2005, 52(9):2854-2864

8. Ruperto N, Ravelli A, Oliveira S, Alessio M, Mihaylova D, Pasic S, et al: The Pediatric Rheumatology International Trials Organization/American College of Rheumatology provisional criteria for the evaluation of response to therapy in juvenile systemic lupus erythematosus: prospective validation of the definition of improvement. Arthritis Rheum 2006, 55(3):355-363.

9. Weening JJ, D'Agati VD, Schwartz MM, Seshan SV, Alpers CE, Appel GB, et al: The classification of glomerulonephritis in systemic lupus erythematosus revisited. J Am Soc Nephrol 2004, 15(2):241-250.

10. Pluchinotta FR, Schiavo B, Vittadello F, Martini G, Perilongo G, Zulian F: Distinctive clinical features of pediatric systemic lupus erythematosus in three different age classes. Lupus 2007, 16(8):550-555.

11. Pattaragarn A, Sumboonnanonda A, Parichatikanond P, Supavekin S, Suntornpoch V, Vongjirad A: Systemic lupus erythematosus in Thai children: clinicopathologic findings and outcome in 82 patients. $J$ Med Assoc Thai 2005, 88(Suppl 8):S232-S241.

12. Al-Mosawi Z, Al-Hermi BE, Al-Saad KK, Farid EM, Makki HA: Juvenile systemic lupus erythematosus in Bahrain. A tertiary referral center experience. Saudi Med J 2009, 30(5):667-672.

13. Gonzalez B, Hernandez P, Olguin H, Miranda M, Lira L, Toso M, et al: Changes in the survival of patients with systemic lupus erythematosus in childhood: 30 years experience in Chile. Lupus 2005, 14(11):918-923.

14. Ramirez Gomez LA, Uribe UO, Osio UO, Grisales Romero H, Cardiel MH, Wojdyla D, et al: Childhood systemic lupus erythematosus in Latin America. The GLADEL experience in 230 children. Lupus 2008, 17(6):596-604.

15. Supavekin S, Chatchomchuan W, Pattaragarn A, Suntornpoch V, Sumboonnanonda A: Pediatric systemic lupus erythematosus in Siriraj Hospital. J Med Assoc Thai 2005, 88(Suppl 8):S115-S123.

16. Bogdanovic R, Nikolic V, Pasic S, Dimitrijevic J, Lipovska-Markovic J, EricMarinkovic J, et al: Lupus nephritis in childhood: a review of 53 patients followed at a single center. Pediatr Nephrol 2004, 19(1):36-44.

17. Ataei N, Haydarpour M, Madani A, Esfahani ST, Hajizadeh N, Moradinejad $\mathrm{MH}$, et al: Outcome of lupus nephritis in Iranian children: prognostic significance of certain features. Pediatr Nephrol 2008, 23(5):749-755.

18. Bakr A: Epidemiology treatment and outcome of childhood systemic lupus erythematosus in Egypt. Pediatr Nephrol 2005, 20(8):1081-1086.

19. Dall'era M, Stone D, Levesque V, Cisternas M, Wofsy D: Identification of biomarkers that predict response to treatment of lupus nephritis with mycophenolate mofetil or pulse cyclophosphamide. Arthritis Care Res (Hoboken ) 2011, 63(3):351-357. doi:10.1002/acr.20397. Epub 2010 Nov 15.

20. Mok CC, Mak A, Chu WP, To CH, Wong SN: Long-term survival of southern Chinese patients with systemic lupus erythematosus: a prospective study of all age-groups. Medicine (Baltimore) 2005, 84(4):218-224.

21. Takei S, Maeno N, Shigemori M, Imanaka H, Mori H, Nerome Y, et al: Clinical features of Japanese children and adolescents with systemic lupus erythematosus: results of 1980-1994 survey. Acta Paediatr Jpn 1997, 39(2):250-256.

22. Gedalia A, Molina JF, Molina J, Uribe O, Malagon C, Espinoza LR: Childhoodonset systemic lupus erythematosus: a comparative study of AfricanAmericans and Latin Americans. J Natl Med Assoc 1999, 91(9):497-501.

doi:10.1186/1546-0096-10-38

Cite this article as: Dung et al: Juvenile systemic lupus erythematosus onset patterns in Vietnamese children: a descriptive study of 45 children. Pediatric Rheumatology 2012 10:38. 